\title{
Design of magnetron co-sputtering configuration for preparing magnesium tin silicide-based thermoelectric alloy thin films
}

\author{
Anh Tuan Thanh Pham ${ }^{1,2}$, Cuong Nhat Le ${ }^{1,2}$, Dung Van Hoang ${ }^{1,2}$, Truong Huu Nguyen ${ }^{1,2}$, \\ Phuong Thanh Ngoc Vo ${ }^{1,2,3}$, Thang Bach Phan ${ }^{1,2,4}$, Vinh Cao Tran ${ }^{1,2}$
}

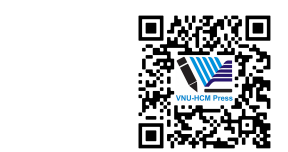

Use your smartphone to scan this QR code and download this article

${ }^{1}$ Laboratory of Advanced Materials,

University of Science, Ho Chi Minh City, Vietnam

${ }^{2}$ Vietnam National University, Ho Chi Minh City, Vietnam

${ }^{3}$ Faculty of Materials Science and Technology, University of Science, Ho Chi Minh City, Vietnam

${ }^{4}$ Center for Innovative Materials and Architectures (INOMAR), Ho Chi Minh City, Vietnam

\section{History}

- Received: 2019-11-16

- Accepted: 2019-12-17

- Published: 2019-12-31

DOI : 10.32508/stdj.v22i4.1729

\section{Check for updates}

\section{Copyright}

(.) VNU-HCM Press. This is an openaccess article distributed under the terms of the Creative Commons Attribution 4.0 International license.

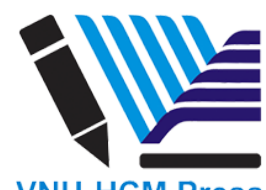

\begin{abstract}
Introduction: Magnesium tin silicide (MgSiSn) is known as a good-thermoelectric-performance, safe and cost-efficient alloy material. The goal of this work is to design a magnetron co-sputtering configuration for depositing alloy thin films from three independent metal targets including magnesium (Mg), silicon (Si) and tin (Sn). Methods: By this solution, the elemental composition of the MgSiSn thin films can be effectively controlled through changing the sputtering power of the individual magnetron. The actual values of elemental composition in the as-deposited films were verified by using energy-dispersive $X$-ray spectroscopy. The as-deposited thin films were investigated carefully by using the $X$-ray diffraction to recognize crystalline structure characteristics. Most importantly, typically thermoelectric parameters including Seebeck coefficient, electrical conductivity and power factor were indicated as functions of temperature. Results: The XRD analysis exhibits cubic anti-fluorite-type structure characteristics of the MgSiSn films; however, the presence of the segregated $\mathrm{Mg}$ phase is still observed. The testing results for the representative MgSiSn thin film with good adherence show the power factor of PF $\sim 20.5 \times 10^{-3} \mathrm{~W} / \mathrm{mK}^{2}$, as a result of Seebeck coefficient of $S \sim 159 \mu \mathrm{V} / \mathrm{K}$ and electrical conductivity of $\sigma \sim 8200 \mathrm{~S} / \mathrm{cm}$, at $325 \mathrm{~K}$. At higher temperature than $473 \mathrm{~K}$, the semiconducting behavior of the films tends to transform from $\mathrm{p}$-type to n-type. Conclusion: The three-target co-sputtering configuration shows the possibility of successfully preparing alloy MgSiSn thin films with good adherence on Si substrate. Furthermore, the testing result suggests that the as-deposited MgSiSn thin films have some potential thermoelectric characteristics, which can be improved more significantly.
\end{abstract}

Key words: Thermoelectrics, magnesium tin silicide, magnetron co-sputtering, thin films

\section{INTRODUCTION}

Magnesium tin silicide (MgSiSn) ternary alloy is one of the best lead-free thermoelectric materials in the medium temperature range $\left(200-600^{\circ} \mathrm{C}\right)$. It has attracted much interest due to constituted composition from the rich-abundant and non-toxic elements ${ }^{1,2}$. According to the estimation expression of thermoelectric figure of merit $(\mathrm{Z}), \mathrm{ZT}=\mathrm{S}^{2} \sigma / \kappa$ (where $\mathrm{S}$ is Seebeck coefficient, $\sigma$ and $\kappa$ are electrical and thermal conductivities, respectively), the increase of $S, \sigma$ values and the reduction of $\kappa$ value result in enhancement of $\mathrm{ZT}$ value. In the case of the MgSiSn alloy, $\mathrm{Si}^{4+}$ replacement of $\mathrm{Sn}^{4+}$ ion not only increases $\mathrm{S}$ value owing to the increasing density of state (DOS) in energy-band structure, but also reduces $\kappa$ value because $\mathrm{Sn}$ atom has much heavier mass than $\mathrm{Si}$ atom ${ }^{3,4}$. Another method to achieve high ZT value is producing low-dimensional materials due to the quantum confinement, high $\sigma$, and low $\kappa$ values ${ }^{5}$. Thin film is known as one of the low-dimensional materials, which doping effect and stoichiometry can be con- trolled. In literature, there have been limited works on the MgSiSn thin films, as compared to the bulk form. Typically, a study on the very thin MgSiSn film (50 - $90 \mathrm{~nm})$ deposited on Si substrate was reported for optoelectronic and thermoelectric applications ${ }^{6}$. The used deposition technique, however, was a solid phase epitaxy (SPE), which is quite a complicated, expensive and hard-to-control method. Recently, the $\mathrm{Al}$ - and $\mathrm{Sn}$-doped $\mathrm{Mg}_{2} \mathrm{Si}$ thin films deposited by using low-cost and high-efficiency sputtering method were attracted $^{7}$. The MgSiSn film was co-sputtered from $\mathrm{Mg}_{2} \mathrm{Si}$ and $\mathrm{Sn}$ targets. It facilitated to adjust the $\mathrm{Sn}$ content. However, the $\mathrm{Mg}$ and $\mathrm{Si}$ contents were not independent because their vapor pressure is very different.

To solve the above problems, in this work, a new cosputtering configuration was set up. The magnetron sputtering system was used to prepare the MgSiSn thin films from three independent metal $(\mathrm{Mg}, \mathrm{Si}$ and Sn) targets. Some electrical and thermoelectric characteristics of the as-deposited MgSiSn thin films were basically investigated. 


\section{MATERIALS - METHODS}

The 3-inch metal targets included $\mathrm{Mg}$, $\mathrm{Si}$, and $\mathrm{Sn}$ (99.99\%, Gredmann, Taiwan) were used to co-sputter the MgSiSn thin films. Because of low conductivity, the Si target was connected to a $13.56 \mathrm{MHz}$ radiofrequency (RF) source, while the Mg and Sn targets were controlled by direct-current (DC) sources. All the MgSiSn films were prepared on a Leybold Univex450 (Germany) sputtering system. The magnetron cosputtering configuration inside vacuum chamber can be modified to change conditional parameters, properties and composition of the films. The $2 \times 2 \mathrm{~cm}^{2}$ $\mathrm{Si}(200)$ wafer was used as substrate. The base vacuum pressure was set at $4 \times 10^{-6}$ torr, which was created by using a high-speed turbomolecular pump. The substrate temperature and working pressure in pure Ar gas atmosphere were maintained at $300^{\circ} \mathrm{C}$ and 3.5 mtorr, respectively. The distance from the target to the substrate was fixed at $7 \mathrm{~cm}$ for all the targets. Before the deposition process, the three targets were presputtered in 5 minutes to remove oxide layers and contamination on the target surface. Also, the substrate was cleaned by discharge in the high-pressure Ar gas atmosphere ( $10^{-2}$ torr).

The deposition time was fixed at 5 minutes corresponding to the film thickness of $\sim 300 \mathrm{~nm}$. The thickness was determined by using a Stylus profilometer (Veeco Dektak-6M, US) and cross-sectional scanning electron microscopy (FESEM, Hitachi S-4800, Japan). In the Stylus method, the Dektak-6M system was equipped a $12.5 \mu \mathrm{m}$ diamond tip. During the measuring process, the Stylus tip contacted and scanned mechanically on the film surface. A height deviation of the tip between the substrate and the film on the substrate was used to derive the film thickness. In the FESEM method, the MgSiSn films on Si substrate were observed horizontally. The obtained cross-sectional image gave information about the crystallization inside the films and the interface between the film and the substrate.

The crystalline structure of the films which was controlled through adjusting the power of the sputtering targets was investigated by using the X-ray diffraction method (XRD, Bruker D8-Advance, US) with a monochromatic $\mathrm{CuK} \alpha$ beam $(\lambda=0.1541 \mathrm{~nm})$ as an $\mathrm{X}$-ray source. In the XRD method, the $\theta-2 \theta$ scanning technique was employed, which $\theta$ is the angle between incident beam and reflected plane, whereas $2 \theta$ is the angle between transmitted beam and reflected beam (detector). While the power of Mg target was fixed at $30 \mathrm{~W}$, the power of Si target increases from 0 to $100 \mathrm{~W}$ corresponding to the decrease of the power of Sn target from 60 to $0 \mathrm{~W}$, as listed in Table 1. The deposition rate from the different metal targets was measured by using a quartz crystal oscillator (Inficon XTM/2, US). In this method, a quartz crystal sensor was applied parallel to the target surface with a similar target-substrate distance $(7 \mathrm{~cm})$. During the sputtering process, the sputtered particle from the targets bombarded on the quartz surface. Owing to piezoelectric property, the vibration resonance of quartz crystal created electrical signals. Based on these recorded signals, the deposition rate from each target was calculated.

The temperature-dependent thermoelectric properties (Seebeck coefficient and electrical conductivity) of the representative MgSiSn thin film was determined by using a Seebeck measurement system (U1vac ZEM-3, Japan). The sample was cut into 15$\mathrm{mm}$ long and 5-mm wide rectangular piece for the measurement. The investigated range and accelerating rate of temperature were $300-675 \mathrm{~K}$ and 50 $\mathrm{K} / \mathrm{min}$, respectively. At each temperature, the values of electrical conductivity and the Seebeck coefficient of the MgSiSn film were measured three times to check the repeatability of the results. In addition, the elemental composition of the representative film was also checked through energy-dispersive X-ray spectroscopy (EDS) which was an attachment of the FESEM technique.

\section{RESULTS}

\section{Design of magnetron co-sputtering config- uration}

To prepare MgSiSn alloy thin films, we modified a co-sputtering configuration with three separate $\mathrm{Mg}$, $\mathrm{Si}$ and Sn targets, which was based on the Leybold Univex-450 system (Figure 1a). The position of the targets was arranged as shown in Figure $\mathbf{1 b}$ and Figure $1 \mathrm{c}$. The sputtering targets were located on the surface of magnetron guns which were continuously cooled at $20^{\circ} \mathrm{C}$ by using a water chiller. Because of the lowest vapor pressure, the sputtering yield of $\mathrm{Mg}$ target is very high. To protect the substrate during the target pre-sputtering process, double shutters were designed. The lower shutter covered the Mg target surface, while the substrate was shielded by the upper shutter. The substrate was attached on the holder which rotates around a centered axis with a rotational angle of $\sim 270^{\circ} \mathrm{C}$ (from $\mathrm{A}$ to $\mathrm{B}$ and vice versa). The holder could rotate continuously with controllable angular velocity. The three magnetron guns were 15 $\mathrm{cm}$ equidistant from each other and $10-\mathrm{cm}$ equidistant from the rotation axis. The sputtering power of 


\begin{tabular}{|c|c|c|}
\hline Samples & Power of Si target (W) & Power of Sn target (W) \\
\hline $\mathrm{Mg}-100 \mathrm{Si}$ & 100 & 0 \\
\hline Mg-90Si-20Sn & 90 & 20 \\
\hline $\mathrm{Mg}-80 \mathrm{Si}-25 \mathrm{Sn}$ & 80 & 25 \\
\hline $\mathrm{Mg}-70 \mathrm{Si}-30 \mathrm{Sn}$ & 70 & 30 \\
\hline $\mathrm{Mg}-60 \mathrm{Si}-35 \mathrm{Sn}$ & 60 & 35 \\
\hline $\mathrm{Mg}-50 \mathrm{Si}-40 \mathrm{Sn}$ & 50 & 40 \\
\hline $\mathrm{Mg}-40 \mathrm{Si}-45 \mathrm{Sn}$ & 40 & 45 \\
\hline Mg-30Si-50Sn & 30 & 50 \\
\hline Mg-60Sn & 0 & 60 \\
\hline
\end{tabular}

each target and the angular velocity of the substrate holder were the most important parameters which affected the uniformity and composition of the MgSiSn thin films. In this initial study, the investigation was focused on changing the sputtering power of each target, thus the angular velocity was fixed at $0.375 \pi \mathrm{rad} / \mathrm{s}$ during the deposition process.

\section{Initial results of the MgSiSn thin films}

Figure 2 shows the crystalline structure of the $\mathrm{Mg}$ SiSn thin films. There are two peaks at $33.18^{\circ}$ and $47.92^{\circ}$ which belong to the (200) and (220) plane of the Si substrate, respectively. A clear peak located at $\sim 34.50^{\circ}$ is found to be the (002) plane of metal $\mathrm{Mg}$ phase (JCPDS 35-0821). The existence of a separate $\mathrm{Mg}$ phase reflects non-uniform stoichiometry or excessive $\mathrm{Mg}$ content in the films. This phenomenon was also reported by Zhang's work ${ }^{7}$. More importantly, it is seen that almost the samples tend to form cubic anti-fluorite-type structure with characteristic crystalline planes, such as (111), (220), (311) and $(222)^{8}$.

Based on the XRD results, the good stoichiometry and low excessive $\mathrm{Mg}$ phase can be obtained in the MgSiSn thin films, if the sputtering power of Si and Sn targets are lower than $60 \mathrm{~W}$ and higher than $35 \mathrm{~W}$, respectively. Among them, the representative Mg-50Si$40 \mathrm{Sn}$ sample is chosen to investigate morphological and thermoelectric properties.

Figure 3 shows the cross-sectional morphology and chemical composition analysis of the Mg-50Si-40Sn thin film. From the FESEM image, the thickness of the film is determined, approximately $300 \mathrm{~nm}$. No layer separation is observed, which suggests good incorporation of the $\mathrm{Mg}$, Si and $\mathrm{Sn}$ contents in the alloy structure. The elemental composition of the film is also checked and listed in the inset table. The EDS result indicates the successful deposition of the alloy MgSiSn film.

Figure 4 shows some typical thermoelectric parameters (electrical conductivity, Seebeck coefficient and power factor) of the Mg-50Si-40Sn thin film. At a lower temperature than $473 \mathrm{~K}$, the electrical conductivity of the films is high, which is highly-degenerated semiconductor behavior. When temperature increases more than $473 \mathrm{~K}$, the electrical conductivity of the films decreases strongly, simultaneously, the value of Seebeck coefficient tends to be more negative. The thermoelectric power factor, $\mathrm{PF}=\mathrm{S}^{2} \sigma$, where $\mathrm{S}$ is the Seebeck coefficient and $\sigma$ is the electrical conductivity. The high PF value means the possibility of generating high voltage and power of thermoelectric materials when there is a temperature gradient. As a result, the highest power factor of PF $\sim 20.5 \times 10^{-3}$ $\mathrm{W} / \mathrm{mK}^{2}$ corresponding to the Seebeck coefficient of $\mathrm{S}$ $\sim 159 \mu \mathrm{V} / \mathrm{K}$ and the electrical conductivity of $\sigma \sim 8200$ $\mathrm{S} / \mathrm{cm}$ can be observed at $\sim 325 \mathrm{~K}$.

\section{DISCUSSION}

Another proof for the formation of MgSiSn alloy is the detection of $\mathrm{Mg}$, Si and $\mathrm{Sn}$ contents in the films, as shown in Figure 3. A problem, however, is that the composition ratio of $\mathrm{Si}$ is very high. It can be due to the contribution of the signals from the Si substrate. Therefore, other materials will be used as a substrate in the future studies. In addition, the $\mathrm{O}$ content may come from residual gas in vacuum chamber or contamination. It is also a technique problem of this co-sputtering configuration for depositing alloy thin films, which is needed to be improved.

From the measurement of thermoelectric properties in Figure 4, the Seebeck coefficient is positive, which 

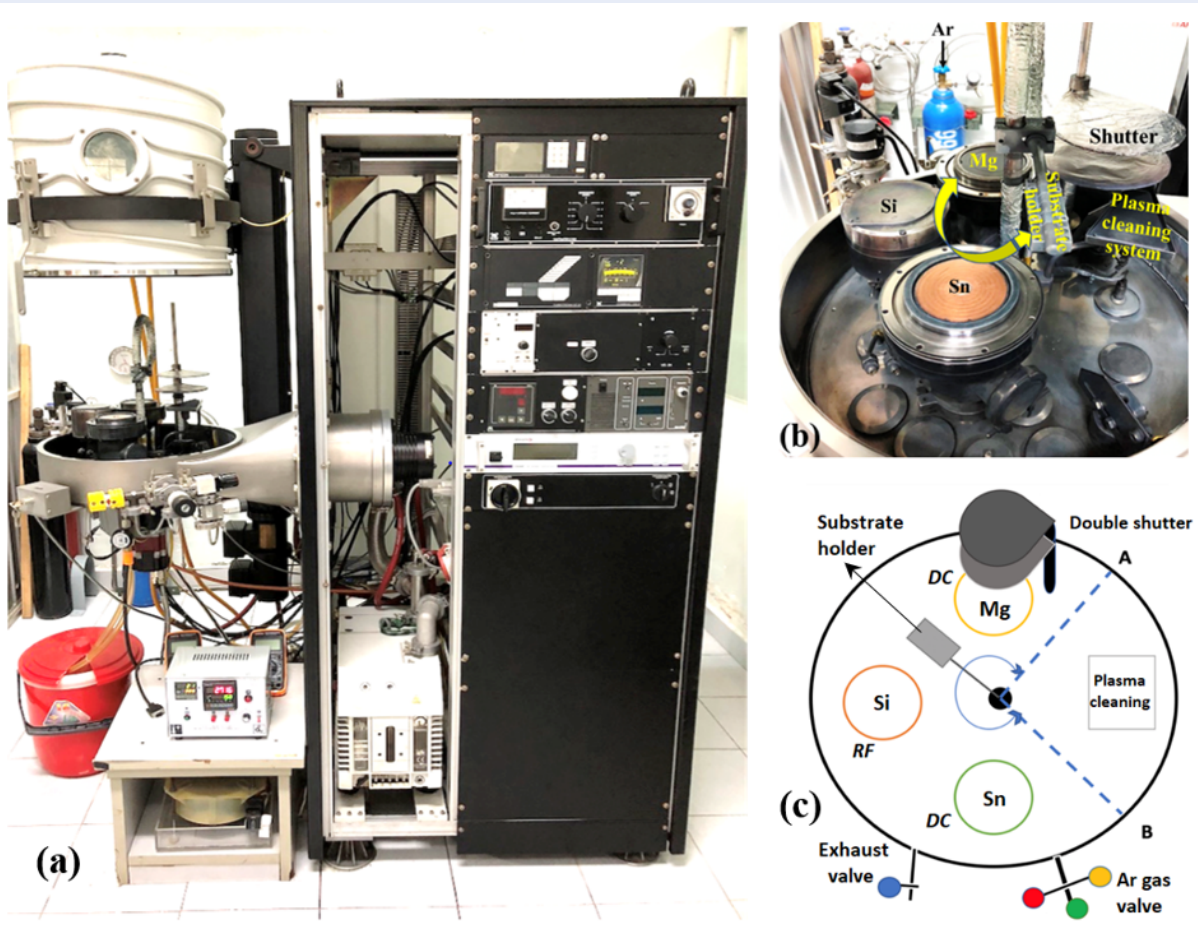

Figure 1: Design of magnetron co-sputtering configuration: (a) Leybold Univex-450 (Germany) sputtering system with high-speed turbomolecular vacuum pump station; (b) and (c) magnetron configuration and targets arrangement in the vacuum chamber. The three targets are equidistant from each other and from the rotation axis of the substrate.

reflects the p-type characteristic of the film. When temperature increases, the electrical conductivity decreases strongly, simultaneously, the film transforms into n-type behavior due to a negative Seebeck coefficient. It may be due to the decrease of carrier concentration and mobility at high temperatures, which is suitable for the characteristic of the highlydegenerated semiconductor. However, the transformation from p-type to n-type behavior of the film has not been understood yet. The obtained PF value is relatively high for the $\mathrm{Mg}_{2}$ Si-based materials, but is still lower than the other reports ${ }^{9-11}$. Consequently, from the above obtained results, the alloy MgSiSn thin films prepared by using the co-sputtering configuration exhibits some thermoelectric properties. Among them, relatively high electrical conductivity and temperature-dependent semiconductor behavior of Seebeck coefficient are interesting. It is believed that the thermoelectric properties of the MgSiSn thin films can be enhanced by optimizing conditional parameters of the co-sputtering configuration.

\section{CONCLUSION}

In conclusion, the three-target co-sputtering configuration shows the possibility of successfully preparing alloy MgSiSn thin films with good adherence on Si substrate. The composition, stoichiometry, crystalline structure and thermoelectric properties of the films can be controlled through adjusting the power sputtering of each target. The typical $300 \mathrm{~nm}$-thick MgSiSn film deposited at $30 \mathrm{~W}$ of $\mathrm{Mg}$ target, $50 \mathrm{~W}$ of Si target and $40 \mathrm{~W}$ of Sn target exhibits the ptype semiconductor behavior with the Seebeck coefficient of $\mathrm{S} \sim 159 \mu \mathrm{V} / \mathrm{K}$, the electrical conductivity of $\sigma \sim 8200 \mathrm{~S} / \mathrm{cm}$ and the power factor of $\mathrm{PF}$ $\sim 20.5 \times 10^{-3} \mathrm{~W} / \mathrm{mK}^{2}$ at $\sim 325 \mathrm{~K}$. The result suggests that the as-deposited MgSiSn thin films have some potential thermoelectric characteristics, which can be improved more significantly in the next studies.

\section{LIST OF ABBREVIATIONS}

$\sigma$ : Electrical conductivity

EDS: Energy-dispersive X-ray spectroscopy

MgSiSn: Magnesium tin silicide

PF: Power factor 


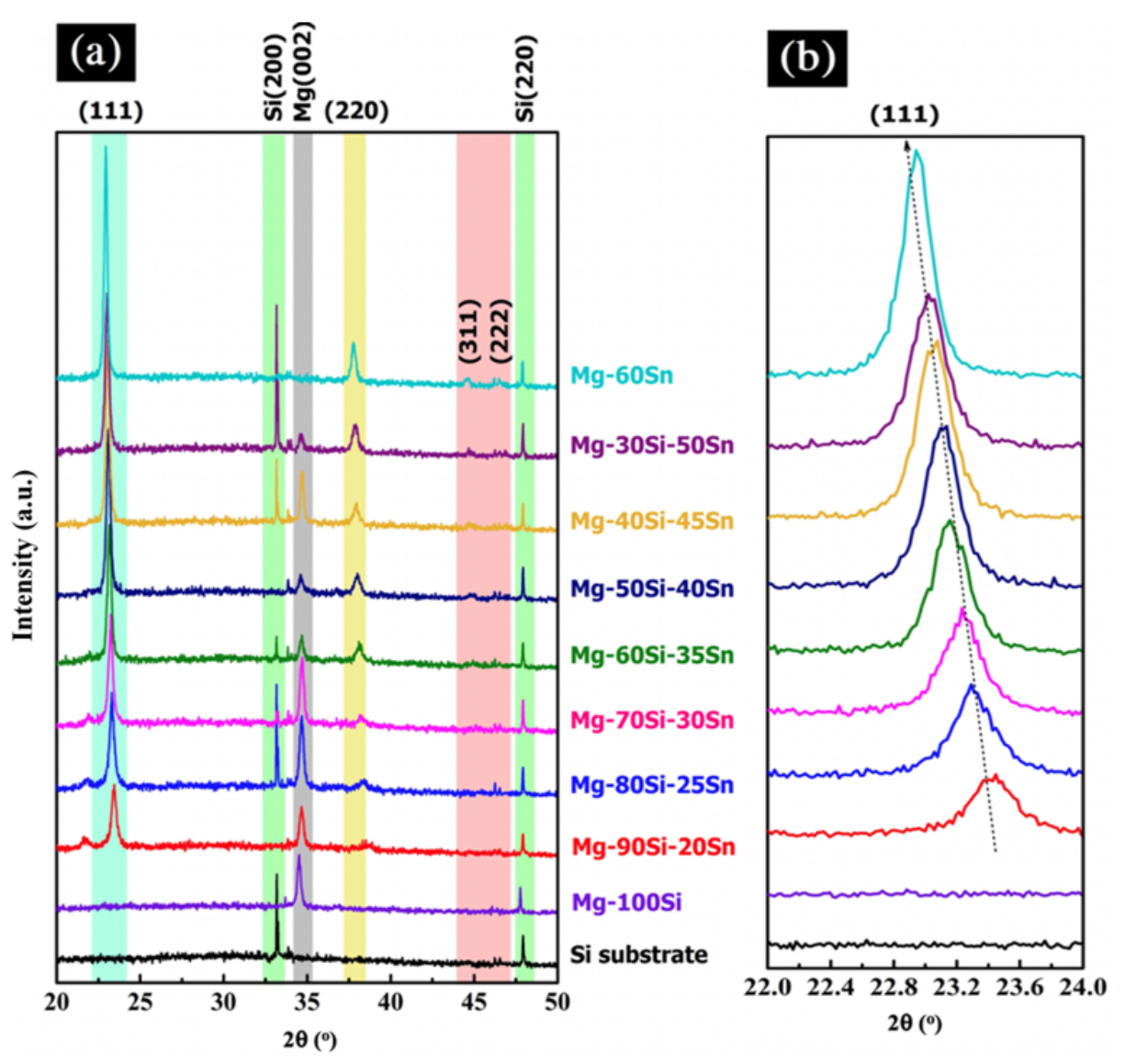

Figure 2: XRD patterns of the MgSiSn thin films deposited with different Si and Sn sputtering powers: (a) in large scale $2 \theta=20-50^{\circ}$, and (b) in small scale $2 \theta=22-24^{\circ}$. The sputtering power of $\mathrm{Mg}$ target is constant, whereas the power of Si target decreases from $100 \mathrm{~W}$ to $0 \mathrm{~W}$, and the power of Sn increases from $0 \mathrm{~W}$ to $60 \mathrm{~W}$.
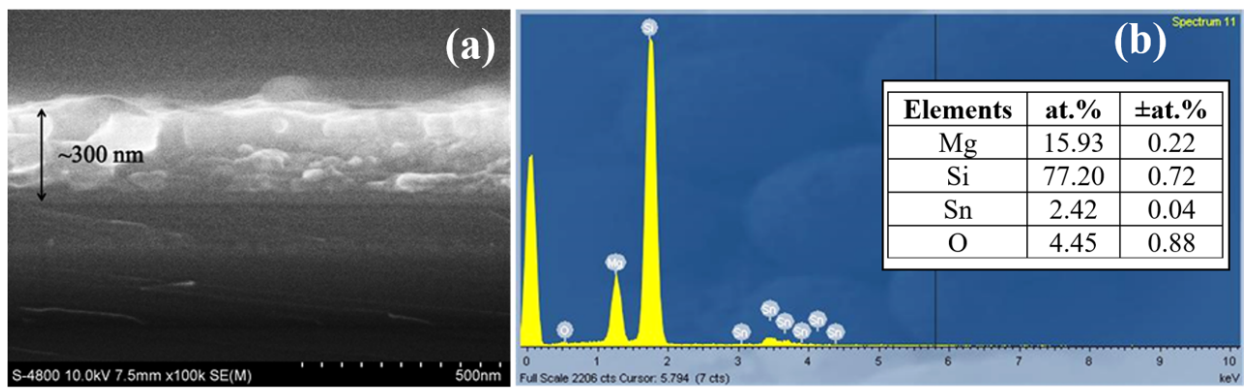

Figure 3: The morphology analysis of the Mg-50Si-40Sn thin film: (a) cross-sectional FESEM image, and (b) EDS elemental quantitative result. The obtained film thickness are about $300 \mathrm{~nm}$, whereas the $\mathrm{O}$ composition might be from contamination. 




Figure 4: Thermoelectric parameters (electrical conductivity, Seebeck coefficient and power factor) of the Mg$50 \mathrm{Si}-40 \mathrm{~S} n$ thin film in the temperature range of $300-675 \mathrm{~K}$.

S: Seebeck coefficient

FE SEM: Field-emission scanning electron microscopy

XRD: X-ray diffraction

\section{COMPETING INTERESTS}

The authors declare that they have no competing interests.

\section{AUTHORS' CONTRIBUTIONS}

All authors of this manuscript have contributed to the work and approved contents of the final version.

\section{ACKNOWLEDGMENTS}

This research is funded by the University of Science, VNU-HCM, under grant number T2018-38.

\section{REFERENCES}

1. Chen HY, Savvides N, Dasgupta T, Stiewe C, Mueller E. Electronic and thermal transport properties of Mg2Sn crystals containing finely dispersed eutectic structures. Phys Status Solidi Appl Mater Sci. 2010;207(11):2523-31. Available from: 10.1002/pssa.201026119.

2. Gao H, Zhu T, Liu X, Chen L, Zhao X. Flux synthesis and thermoelectric properties of eco-friendly Sb doped Mg2Si0.5Sn0.5 solid solutions for energy harvesting. J Mater Chem. 2011;21(16):5933. Available from: 10.1039/c1jm00025j.

3. Luo W, Yang $M$, Chen F, Shen $Q$, Jiang $H$, Zhang L. Fabrication and thermoelectric properties of $M g 2 \operatorname{Si} 1-x \operatorname{Snx}(0 \leq x \leq 1.0)$ solid solutions by solid state reaction and spark plasma sintering. Mater Sci Eng B. 2009;157(1):96-100. Available from: 10.1016/j.mseb.2008.12.029.

4. Liu W, Chi H, Sun H, Zhang Q, Yin K, Tang $X$, et al. Advanced thermoelectrics governed by a single parabolic band: Mg2Si(0.3)Sn(0.7), a canonical example. Phys Chem Chem Phys. 2014;16(15):6893-7. PMID: 24599570. Available from: $10.1039 / \mathrm{c} 4 \mathrm{cp} 00641 \mathrm{k}$.

5. Dresselhaus MS, Chen G, Tang MY, Yang RG, Lee H, Wang $D Z$, et al. New Directions for low-dimensional thermoelectric materials. Adv Mater. 2007;19(8):1043-53. Available from: 10.1002/adma.200600527.

6. Galkin NG, Galkin KN, Dotsenko S, Chernov I, Maslov A, Dózsa L, et al. Mg2SixSn1-x heterostructures on Si(111) substrate for optoelectronics and thermoelectronics. Proc SPIE. 2016;10176(111):1017604. Available from: 10.1117/12. 2268266.

7. Zhang B, Zheng T, Sun C, Guo Z, Kim MJ, Alshareef HN, et al. Electrical transport characterization of Al and Sn doped Mg2Si thin films. J Alloys Compd. 2017;720:156-60. Available from: 10.1016/j.jallcom.2017.05.224.

8. Morozova NV, Ovsyannikov SV, Korobeinikov IV, Karkin AE, Takarabe K, Mori Y, et al. Significant enhancement of thermoelectric properties and metallization of Al-doped Mg2Si under pressure. J Appl Phys. 2014;115(21):213705. Available from: 10.1063/1.4881015.

9. Abe R, Fujishiro $H$, Naito T. Substitution effect of tetravalent and pentavalent elements on thermoelectric properties in In2O3-SnO2 system. Trans Mater Res Soc Jpn. 2016;41(1):1018. Available from: 10.14723/tmrsj.41.101.

10. Zaitsev VK, Fedorov MI, Eremin IS, Gurieva EA. Rowe DM, editor. Thermoelectrics on the base of solid solutions of Mg2BIVCompounds (BIV = Si, Ge, Sn). CRC Taylor \&amp; Francis; 2006.

11. Jiang G, He J, Zhu T, Fu C, Liu X, Hu L, et al. High performance $\mathrm{Mg} 2(\mathrm{Si}, \mathrm{Sn})$ solid solutions: a point defect chemistry approach to enhancing thermoelectric properties. Adv Funct Mater. 2014;24(24):3776-81. Available from: 10.1002/adfm. 201400123. 